EPJ Web of Conferences 63,02013 (2013)

DOI: 10.1051/epjconf/ 20136302013

(C) Owned by the authors, published by EDP Sciences, 2013

\title{
In-beam Fission Study at JAEA
}

\author{
Katsuhisa Nishio ${ }^{1, a}$ \\ ${ }^{1}$ Advanced Science Research Center, Japan Atomic Energy Agency, Tokai, Ibaraki 319-1195, Japan.
}

\begin{abstract}
.
Fission fragment mass distributions were measured in heavy-ion induced fissions using ${ }^{238} \mathrm{U}$ target nucleus. The measured mass distributions changed drastically with incident energy. The results are explained by a change of the ratio between fusion and quasifission with nuclear orientation. A calculation based on a fluctuation dissipation model reproduced the mass distributions and their incident energy dependence. Fusion probability was determined in the analysis. Evaporation residue cross sections were calculated with a statistical model in the reactions of ${ }^{30} \mathrm{Si}+{ }^{238} \mathrm{U}$ and ${ }^{34} \mathrm{~S}+{ }^{238} \mathrm{U}$ using the obtained fusion probability in the entrance channel. The results agree with the measured cross sections for seaborgium and hassium isotopes.
\end{abstract}

\section{Introduction}

Experiments to produce superheavy nuclei (SHN) have been carried out by using heavy-ion fusion and evaporation reactions [1-3]. Prediction of the cross sections for $\mathrm{SHN}$ is important to make an experimental plan and explore this region of chart of nuclei. The reaction proceeds in three steps; (1) penetration of the Coulomb barrier between two colliding nuclei (capture), (2) formation of a compound nucleus after nuclear contact (3) survival of the excited compound nucleus by particle evaporation against fission. The first step, penetrating the Coulomb barrier, is relatively well understood. Survival probability of compound nucleus can be calculated in a statistical model. The second process, forming a compound nucleus (fusion probability, $P_{\text {fus }}$ ), is not well understood, and to understand this process is the subject of this research program.

We studied fusion reactions using ${ }^{238} \mathrm{U}$ target nucleus. The reactions using actinide-target nuclei have been extensively used for the heavy element synthesis. These reactions produce more neutron rich SHN than those using the cold fusion reactions, and their decay properties have information on the structure in the vicinity of spherically closed-shell at $N=184, Z=114(120,126)$. Some of the isotopes have a half-life long enough to study chemical properties. Actinide nuclei have a prolate deformation, which should influence the fusion probability. At the collision on the polar sides the Coulomb barrier is low, and the reaction starts from a distant configuration. Collision on the equatorial side has higher Coulomb barrier. We studied the orientation effects on fusion and/or quasifission by measuring the fission fragment mass distributions. With the analysis using fluctuation dissipation model, fusion probability is determined. Validity of the proposed method to determine

\footnotetext{
${ }^{\mathrm{a}} \mathrm{e}-\mathrm{mail}$ : nishio.katsuhisa @ jaea.go.jp
}

fusion probability was confirmed by measuring the evaporation residue cross sections for seaborgium and hassium isotopes produced in the ${ }^{30} \mathrm{Si}+{ }^{238} \mathrm{U}$ and ${ }^{34} \mathrm{~S}+{ }^{238} \mathrm{U}$ reactions, respectively.

\section{Experimental Methods}

Fission fragment mass distributions in the reactions of ${ }^{30} \mathrm{Si},{ }^{31} \mathrm{P},{ }^{34,36} \mathrm{~S},{ }^{40} \mathrm{Ar},{ }^{40,48} \mathrm{Ca}+{ }^{238} \mathrm{U}$ were measured using beams supplied by the tandem accelerator of the Japan Atomic Energy Agency (JAEA) at Tokai. The experimental set-up and the analysis method were described in [4]. The beam intensities were typically from 0.1 to $1.0 \mathrm{p}$-nA. The ${ }^{238} \mathrm{U}$ target was prepared by electrodeposition of $\mathrm{UO}_{2}$ on a $90 \mu \mathrm{g} / \mathrm{cm}^{2}$ thick nickel backing. The thickness of ${ }^{238} \mathrm{U}$ was about $80 \mu \mathrm{g} / \mathrm{cm}^{2}$. Both fission fragments (FFs) were detected in coincidence by position-sensitive multiwire proportional counters (MWPCs) having an active area of $200 \mathrm{~mm}(\mathrm{H}) \times 120 \mathrm{~mm}(\mathrm{~V})$. The detectors were located on both sides of the target at a distance of $211 \mathrm{~mm}$. The detector center was placed to optimize the efficiency to detect fission fragment in coincidence. The MWPCs covered the emission angle of $\pm 25.0^{\circ}$ around the detector center.

The time difference, $\Delta T$, between the signals from the cathodes of MWPC1 and MWPC2 was measured. The charges induced in two MWPCs contain information on the energy deposition $\Delta E_{1}$ and $\Delta E_{2}$ of particles traversing the detectors and were recorded.

Fission events occurring after complete transfer of the projectile momentum to the composite system (full momentum transfer (FMT) fission) were separated from those fission events following nuclear transfer by recording the folding angle formed by two fission fragments. Details of the data analysis are shown in $[4,10,11]$. 


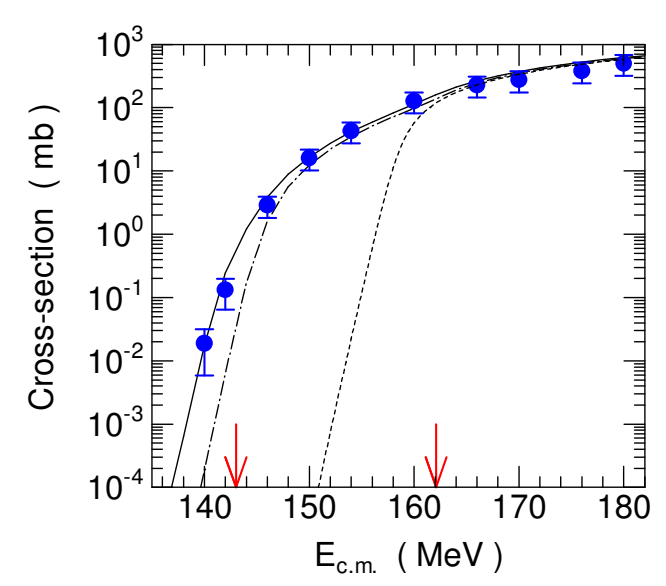

Figure 1. Cross-sections for the full momentum transfer (FMT) fission of the reaction ${ }^{36} \mathrm{~S}+{ }^{238} \mathrm{U}$. Curves represent the results of coupled-channel calculations (see text). The Coulomb barriers for polar and equatorial collisions are at 143.0 and $162.1 \mathrm{MeV}$, respectively, as indicated by the arrows.

\section{Experimental Results and Discussions}

The cross-sections for the FMT fissions $\left(\sigma_{\text {fiss }}\right)$ in the reaction ${ }^{36} \mathrm{~S}+{ }^{238} \mathrm{U}$ are shown in Fig. 1 as a function of centerof-mass energy $E_{\mathrm{cm}}$.

The cross-sections are almost equal to those of the projectiles being captured inside the Coulomb barrier $\left(\sigma_{\text {cap }}\right)$. In order to see the influence of nuclear properties on the capture cross-sections, we performed a coupled-channel calculation using the computer code CCDEGEN [5]. We used the same parameters for the nuclear potential as in our previous work for the reactions ${ }^{16} \mathrm{O}+{ }^{238} \mathrm{U}$ [6]. The dashed curve in Fig. 1 is the result without considering any collective properties of target and projectile (one-dimensional barrier penetration model). This model does not reproduce the cross-sections for $E_{\mathrm{cm}}<160 \mathrm{MeV}$. The dashdotted curve represents the calculation taking into account the deformation of ${ }^{238} \mathrm{U}$ with $\beta_{2}=0.275$ and $\beta_{4}=0.05$ $[6,7]$. These results reproduce the data well down to $E_{\mathrm{cm}}=146.0 \mathrm{MeV}$, showing that the static deformation of ${ }^{238} \mathrm{U}$ is the main reason for the cross-section enhancement at sub-barrier energies. Data at the two lowest energies of 142.0 and $140 \mathrm{MeV}$ are reproduced when couplings to vibrational states are additionally taken into account (solid curve), where couplings to the $2^{+}$state at $3.29 \mathrm{MeV}$ in ${ }^{36} \mathrm{~S}$ $\left(\beta_{2}=0.16\right.$ [8] ) and the $3^{-}$state at $0.73 \mathrm{MeV}$ in ${ }^{238} \mathrm{U}($ $\beta_{3}=0.086$ [9] ) were considered.

The measured fission fragment mass distributions in the reactions of ${ }^{30} \mathrm{Si},{ }^{31} \mathrm{P},{ }^{36} \mathrm{~S},{ }^{40} \mathrm{Ar},{ }^{48} \mathrm{Ca}+{ }^{238} \mathrm{U}$ are shown in Fig.2 [10-12]. In each reaction, data at four incident energy points are shown. In the ${ }^{30} \mathrm{Si},{ }^{31} \mathrm{P}$ and ${ }^{36} \mathrm{~S}$-induced reactions, we observed a mass-symmetric distribution at the highest incident energy. The mass-asymmetric fission channel appears at the low energies. The variation of the measured distributions with incident energy is interpreted by the effects of nuclear orientation on fusion and/or quasifission. At the lowest incident energies, the reaction is

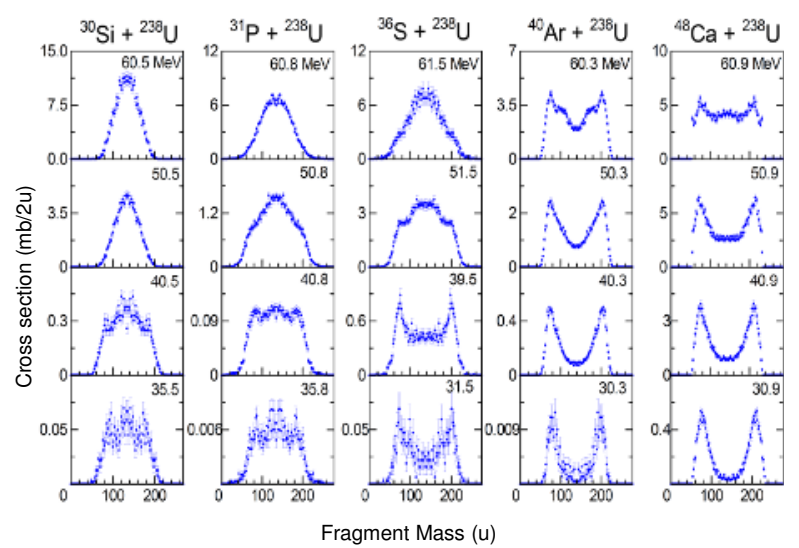

Figure 2. Fission fragment mass distributions of full momentum transfer fission. The numerical value in each section of the figure show the excitation energy of the compound nucleus.

limited to the collision on the polar sides of the nucleus ${ }^{238} \mathrm{U}$. This configuration leads to quasifission with higher probability than the reaction starting from equatorial collisions. It is evident from Fig. 2 that quasifission probability increases with the mass and/or charge of projectile nucleus. In the reactions using ${ }^{40} \mathrm{Ar}$ and ${ }^{48} \mathrm{Ca}$ beams, the mass-asymmetric quasifission dominates for all incident energies. However, fraction of the symmetric-fission increases with incident energy, showing the orientation effects.

In order to make a quantitative analysis of the mass distribution and to determine the fusion probability $P_{\text {fus }}$, we performed a model calculation combining the coupled channels method and a dynamical description of the reaction based on the three-dimensional Langevin equation [13]. The dynamical calculation based on the Monte Carlo method was used for describing the reaction paths in the potential energy landscape. Potential energy surface was calculated with the two-center shell model, and three parameters, charge-center distance, mass asymmetry and deformation, were chosen to describe the nuclear shape. The calculation started at the contact configuration. The deformation of the reaction partners and their statistical orientation in the reaction plane was considered. The coupled channels method was first used to compute the penetration probability of the Coulomb barrier for a fixed orientation angle. The dynamical calculation was then started from the shape at contact configuration for each orientation. In this model, evolution of nuclear shape from the nuclear contact point is tracked with time by solving the Langevin equations and trajectories were calculated down to the scission point. Fusion is defined as the case when a compound nucleus is formed. Quasifission is the binary decay without reaching the compound nucleus shape.

Figure 3 shows a time evolution of calculated probability distribution plotted on the charge-center distance and mass asymmetry in the reactions of ${ }^{30} \mathrm{Si}+{ }^{238} \mathrm{U}$ and ${ }^{36} \mathrm{~S}+{ }^{238} \mathrm{U}$ [13]. The excitation energy of the former and the latter calculations are $35.5 \mathrm{MeV}$ and $39.5 \mathrm{MeV}$, respec- 


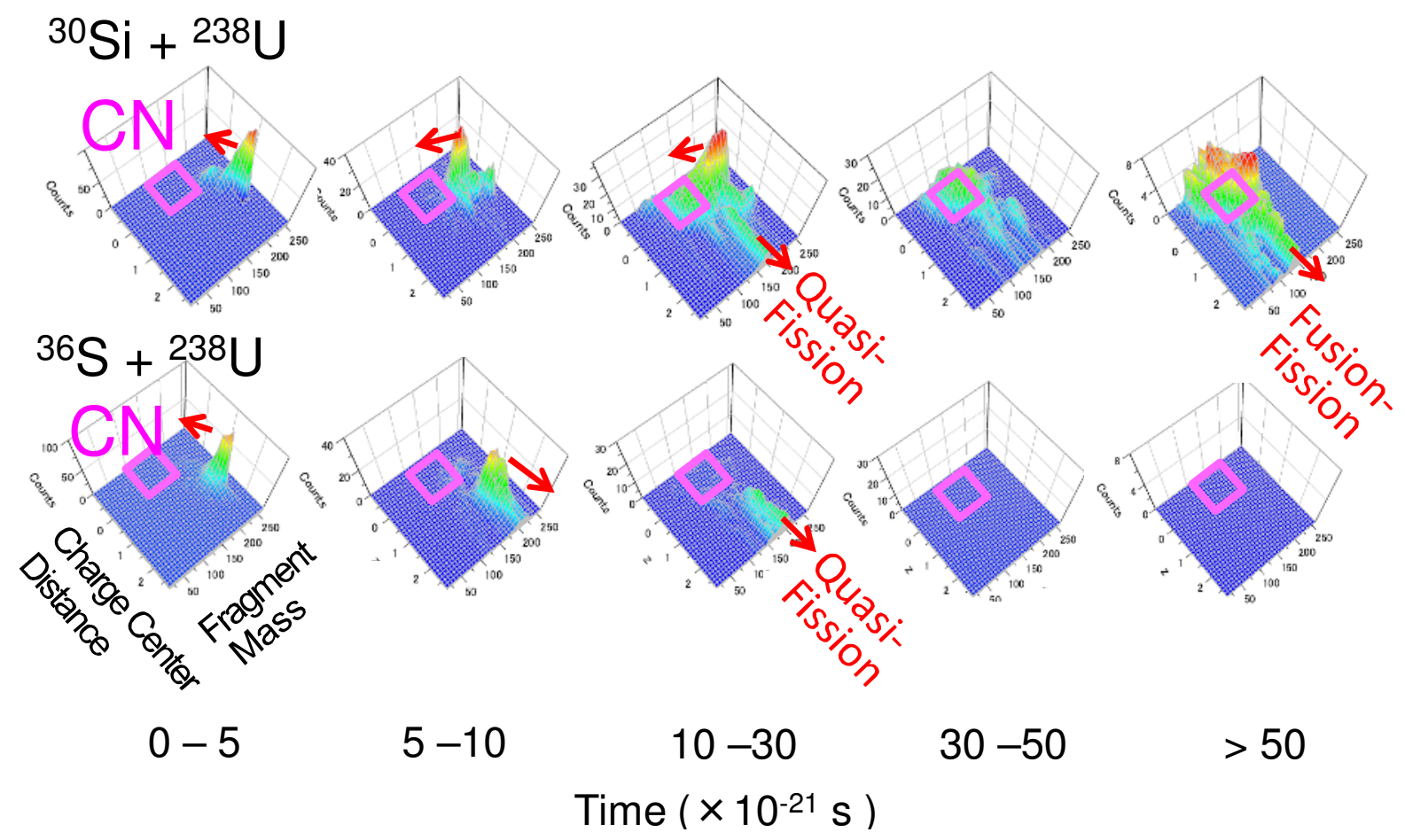

Figure 3. Probability distributions of the reaction ${ }^{30} \mathrm{Si}+{ }^{238} \mathrm{U}$ and ${ }^{36} \mathrm{~S}+{ }^{238} \mathrm{U}$ calculated with the fluctuation-dissipation model. The region of compound nucleus shape is marked. Details are shown in [13].

tively, and collisions on the polar sides were assumed. At the early stage of the reaction, $t<5 \times 10^{-21} \mathrm{~s}$, both systems are headed for the compound nucleus shape $(\mathrm{CN})$. The difference, however, appears at the next time interval, $t=5 \sim 10 \times 10^{-21} \mathrm{~s}$. Majority of the system is going to re-separate by quasifission in the ${ }^{36} \mathrm{~S}+{ }^{238} \mathrm{U}$ reaction, whereas the system ${ }^{30} \mathrm{Si}+{ }^{238} \mathrm{U}$ are still moving toward the compound nucleus. In the reaction of ${ }^{30} \mathrm{Si}+{ }^{238} \mathrm{U}$, fission from a compound nucleus shape appears in the later time of $t>50 \times 10^{-21} \mathrm{~s}$.

We compared the measured mass distributions with the model calculation in the reactions of ${ }^{30} \mathrm{Si}+{ }^{238} \mathrm{U}$ and ${ }^{34} \mathrm{~S}$ $+{ }^{238} \mathrm{U}$. The results are shown in Fig.4. Good agreement is found at entire energy range for both reactions. Especially, appearance of the asymmetric fission at the low incident energies is well demonstrated. Fusion-fission events were chosen in the trajectory analysis, and the corresponding mass distributions are also shown in Fig.4. Fusion-fission spectrum shows the mass-symmetric shape, and the shapes are nearly the same for the two compound nucleus, ${ }^{268} \mathrm{Sg}$ and ${ }^{272} \mathrm{Hs}$. Fusion probability $P_{\text {fus }}$ is obtained from the fraction of fusion-fission events. In the reaction of ${ }^{30} \mathrm{Si}+$ ${ }^{238} \mathrm{U}, P_{\text {fus }}$ decreases from $46 \%$ to $29 \%$ toward the low incident energy. The probability changes from $15 \%$ to $3.6 \%$ in the ${ }^{34} \mathrm{~S}+{ }^{238} \mathrm{U}$ reaction. From the spectra, it is found that the mass-symmetric fission fragments do not necessarily originate from fusion-fission, especially for ${ }^{34} \mathrm{~S}$ $+{ }^{238} \mathrm{U}$. The symmetric fission fragments without forming the compound nucleus was interpreted as the deep-

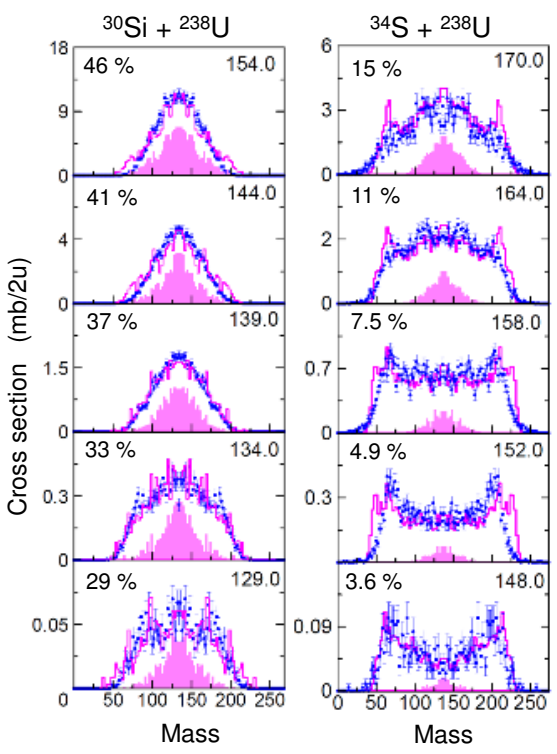

Figure 4. Fission fragment mass distributions for ${ }^{30} \mathrm{Si}+{ }^{238} \mathrm{U}$ and ${ }^{34} \mathrm{~S}+{ }^{238} \mathrm{U}$ are compared with the model calculation. Numerical value marked at the right-top position is the center-of-mass energy. The histogram includes all fission fragments in the calculation. Fusion-fission spectra in the calculation are shown by filled histogram. Fusion probabilities are shown in $\%$ for each energy points. 


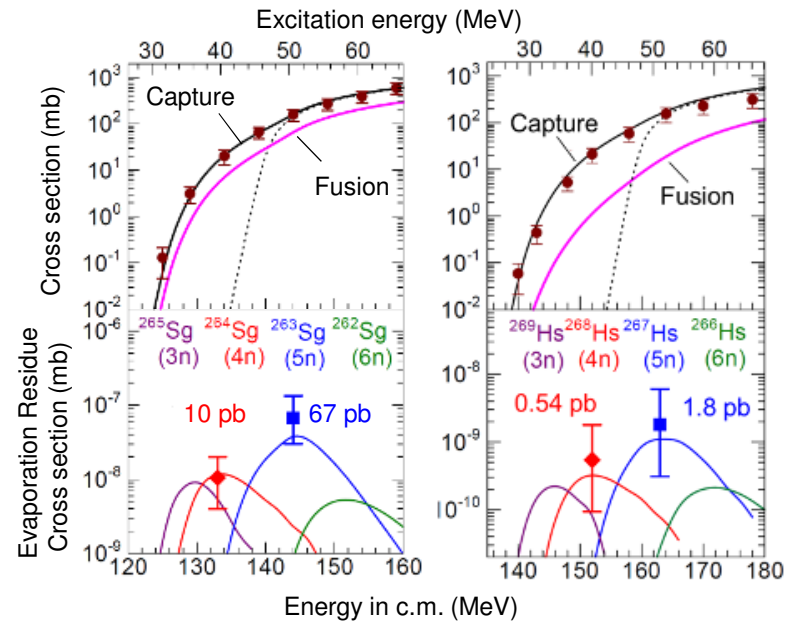

Figure 5. (Upper panel) Measured fission cross sections of the reaction ${ }^{30} \mathrm{Si}+{ }^{238} \mathrm{U}$ (left) and ${ }^{34} \mathrm{~S}+{ }^{238} \mathrm{U}$ (right) as a function of the center-of-mass energy and the excitation energy $\left(E^{*}\right)$ of the compound nucleus. Solid curve (black) show the capture cross-section calculated by the coupled-channels model. Dotted curve is the calculation using one-dimensional barrier penetration model. Solid curve (magenta) is the fusion cross section determined by multiplying the fusion probability to the capture cross section. (Lower panel) Measured cross sections for production of ${ }^{263,264} \mathrm{Sg}$ and ${ }^{267,268} \mathrm{Hs}$. Curves represent results of statistical model calculations for $3 n$ to $6 n$ evaporation channels using the fusion cross sections on the upper panel.

quasifission [14]. In the mass-asymmetric quasifission, system starts to disintegrate soon after the nuclear contact, and the mass-asymmetry parameter of nuclear shape does not change significantly. In the deep-quasifission, the trajectory approaches a nuclear shape with mass-symmetry, but the charge center distance does not reach the region of the compound nucleus.

Validity of the obtained fusion probability was confirmed by measuring the evaporation residue cross sections. Experiment to produce seaborgium isotopes in the reaction of ${ }^{30} \mathrm{Si}+{ }^{238} \mathrm{U}$ was carried out at GSI in Darmstadt [15]. The velocity filter SHIP [2] was used to separate evaporation residues from the primary beams and other reaction products. We produced the isotope ${ }^{263} \mathrm{Sg}$ ( $5 n$ channel) at $E_{\mathrm{cm}}=144.0 \mathrm{MeV}$, which is higher than the Coulomb barrier at the equatorial collision. Cross section of $67 \mathrm{pb}$ was obtained. At the sub-barrier energy of 133.0 MeV, we produced ${ }^{263} \mathrm{Sg}(4 n)$, and the cross section $10 \mathrm{pb}$ was obtained. Figure 5 (left) summarizes the capture cross sections, fusion cross sections and evaporation residue cross sections for ${ }^{30} \mathrm{Si}+{ }^{238} \mathrm{U}$. The cross sections for ${ }^{263,264} \mathrm{Sg}$ were compared with a statistical model calculation, where the obtained fusion probabilities were used as input for the HIVAP code [16]. It is evident from Fig.5 that the measured cross sections agree with the calculation.

The reaction ${ }^{34} \mathrm{~S}+{ }^{238} \mathrm{U}$ was used to produce the hassium isotopes, ${ }^{267,268} \mathrm{Hs}$ [11]. At the incident energy of $E_{\mathrm{cm}}=163.0 \mathrm{MeV}$, we observed one decay chain starting

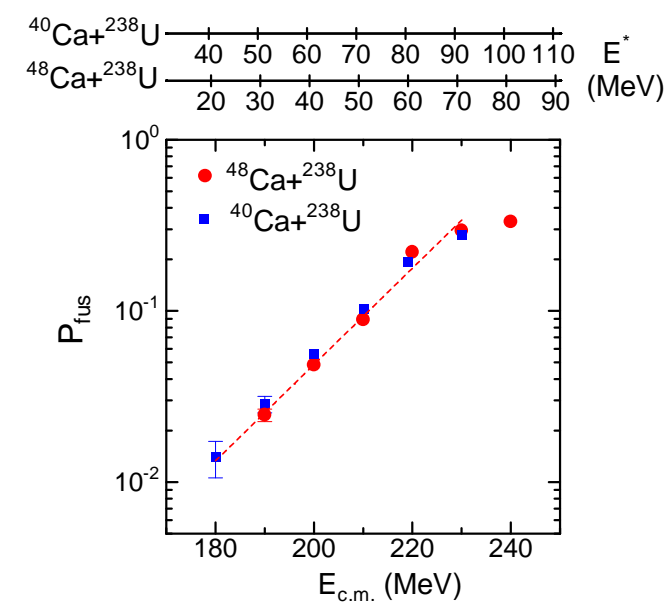

Figure 6. Fusion probabilities in the reactions of ${ }^{40,48} \mathrm{Ca}+{ }^{238} \mathrm{U}$ as a function of center-of-mass energy. The upper panel shows the excitation energy of compound nucleus.

from $5 n$ evaporation residue ${ }^{267} \mathrm{Hs}$. The cross section 1.8 $\mathrm{pb}$ was obtained. At the sub-barrier energy of $152.0 \mathrm{MeV}$, we observed a decay of the new isotope ${ }^{268} \mathrm{Hs}(4 n)$, and the cross section $0.54 \mathrm{pb}$ was obtained. Cross sections are summarized for ${ }^{34} \mathrm{~S}+{ }^{238} \mathrm{U}$ in Fig.5 (right). Compared to the ${ }^{30} \mathrm{Si}+{ }^{238} \mathrm{U}$ reaction, fusion cross sections have smaller values for ${ }^{34} \mathrm{~S}+{ }^{238} \mathrm{U}$. This results in the smaller cross sections for hassium isotopes in the calculation, and the calculation reproduces the measured ER cross sections. It should be also mentioned that the sub-barrier reaction can be also used for the heavy element synthesis. Our analysis suggests the possible production of $3 n$ cannel at the deep sub-barrier energies with the similar cross section to the $4 n$ channel.

Fusion probabilities for ${ }^{40,48} \mathrm{Ca}$ are shown in Fig.6. The probability increases exponentially with incident energy up to $E_{\mathrm{cm}}=230 \mathrm{MeV}$, and the two reactions have identical values. In Fig.6, excitation energies of compound nuclei are also shown. Because of the different $Q$-values, $-138.6 \mathrm{MeV}$ for ${ }^{40} \mathrm{Ca}+{ }^{238} \mathrm{U}$ and $-159.1 \mathrm{MeV}$ for ${ }^{48} \mathrm{Ca}+{ }^{238} \mathrm{U}$, the excitation energy has the difference of $20.5 \mathrm{MeV}$ at a certain incident energy $E_{\mathrm{cm}}$. This necessitate additional two neutron emission for ${ }^{40} \mathrm{Ca}+{ }^{238} \mathrm{U}$ at a certain $E_{\mathrm{cm}}$ to produce evaporation residue, which should reduce the cross sections significantly. The copernicium isotopes are produced in the reaction ${ }^{48} \mathrm{Ca}+{ }^{238} \mathrm{U}$ at $E_{\mathrm{cm}}=190 \sim 200 \mathrm{MeV}[17,18]$, and the corresponding fusion probability is in the range of 0.025 to 0.05 . In the analysis, we expect that $2 n$ evaporation channels could be possible to observe with the maximum cross sections at $E_{\mathrm{cm}}=184 \mathrm{MeV}\left(P_{\text {fus }}=0.017\right)$.

\section{Acknowledgement}

Special thanks are due to the staff of JAEA Tandem facility for supplying heavy-ion beams. The experiments are carried out under the collaboration 
with JAEA, Helmholtzzentrum für Schwerionenforschung (GSI), Flerov Laboratory of Nuclear Reactions, Tohoku University, Technische Universität München, and High Energy Accelerator Organization (KEK).

\section{References}

[1] Yu. Ts. Oganessian, J. Phys. G 34, R165 (2007).

[2] S. Hofmann and G.Munzenberg, Rev. Mod. Phys. 72, 733 (2000).

[3] K. Morita et al., J. Phys. Soc. Jpn. 81, 103201 (2012).

[4] K. Nishio et al., Phys. Rev. C 77, 064607 (2008).

[5] K. Hagino et al., Computer Phys. Comm. 123, 143 (1999).

[6] K. Nishio et al., Phys. Rev. Lett. 93, 162701 (2004).

[7] D.J. Hinde et al., Phys. Rev. Lett., 74, 1295 (1995).
[8] S. Raman et al., At. Data Nucl. Data Tables, 36, 1 (1987).

[9] R.H. Spear et al., At. Data Nucl. Data Tables, 42, 55 (1989).

[10] K. Nishio et al., Phys. Rev. C 82, 044604 (2010).

[11] K. Nishio et al., Phys. Rev. C 82, 024611 (2010).

[12] K. Nishio et al., Phys. Rev. C 86, 034608 (2012).

[13] Y. Aritomo et al., Phys. Rev. C 85, 044614 (2012).

[14] Y. Aritomo and M. Ohta , Nucl. Phys. A753, 152 (2005).

[15] K. Nishio et al., Eur. Phys. J. A 29, 281 (2006).

[16] W. Reisdorf and M. Schädel, Z. Phys. A 343, 47 (1992).

[17] Yu.Ts. Oganessian et al., Phys. Rev. C 70, 064609 (2004).

[18] S. Hofmann et al., Eur. Phys. J. A 32, 251 (2007). 